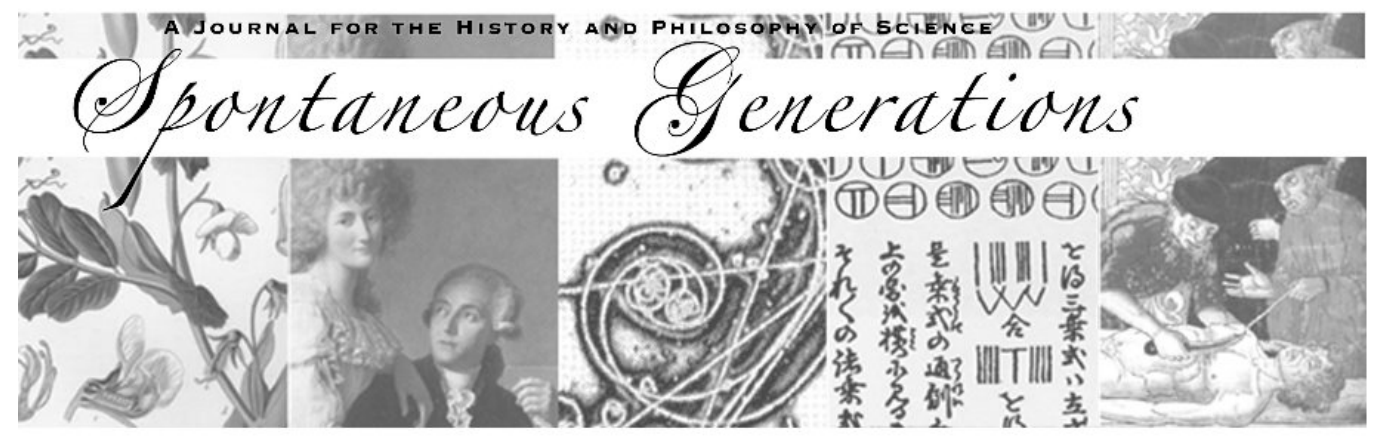

An Instrument for What? Digital Computers, Simulation and Scientific Practice

Author(s): Wendy S. Parker

Source: Spontaneous Generations: A Journal for the History and Philosophy of Science, Vol. 4, No. 1 (2010) 39-44.

Published by: The University of Toronto

DOI: $10.4245 /$ sponge.v4i1.13765

EDITORIAL OFFICES

Institute for the History and Philosophy of Science and Technology

Room 316 Victoria College, 91 Charles Street West

Toronto, Ontario, Canada M5S $1 \mathrm{~K} 7$

hapsat.society@utoronto.ca

Published online at jps.library.utoronto.ca/index.php/SpontaneousGenerations ISSN 19130465

Founded in 2006, Spontaneous Generations is an online academic journal published by graduate students at the Institute for the History and Philosophy of Science and Technology, University of Toronto. There is no subscription or membership fee. Spontaneous Generations provides immediate open access to its content on the principle that making research freely available to the public supports a greater global exchange of knowledge. 


\title{
An Instrument for What? \\ Digital Computers, Simulation and Scientific Practice*
}

\author{
Wendy S. Parker ${ }^{\dagger}$
}

\begin{abstract}
As a device used by scientists in the course of performing research, the digital computer might be considered a scientific instrument. But if so, what is it an instrument for? This paper explores a number of answers to this question, focusing on the use of computers in a simulating mode.
\end{abstract}

If devices used by scientists in the course of performing research qualify as scientific instruments, then it seems clear that a new and particularly important scientific instrument arrived on the scene in the second half of the twentieth century: the digital computer. Indeed, this instrument has proven to be transformative, significantly changing how science is done in many fields. But what sort of instrument is the digital computer? That is, what is it an instrument for? Some scientific instruments-like optical telescopes-serve to enhance our natural perceptual abilities. Other scientific instruments help us carry out desired interventions; we might use a "gene gun" to insert genetic material into plant cells. Still other instruments are for measuring, as in the case of thermometers and Geiger counters. When we consider the digital computer, however, specifying what it is an instrument for is more complicated. ${ }^{1}$

One option is to characterize the digital computer as an instrument for computation. In some sense, this is surely right. As Paul Humphreys suggests in the opening pages of Extending Ourselves, we can view the digital computer as an instrument that enhances our natural computational abilities, much like the optical telescope enhances our natural perceptual abilities (Humphreys 2004, 3-5). With the help of today's computers, we can carry out mathematical operations too complex or lengthy to perform in our heads, and at speeds tremendously faster than we could ever hope

* Received 3 June 2010.

$\dagger$ Wendy S. Parker is Assistant Professor of Philosophy at Ohio University. Her research focuses on the epistemology of computer simulation, especially weather and climate simulation.

1 Though I speak of "the" digital computer, I mean this to include both personal computers (PCs) and more powerful supercomputers of the last several decades. 
to achieve using pencil and paper. Yet simply characterizing the digital computer as an instrument for computation seems unsatisfying, given the variety of uses to which it is put in science. Humphreys himself calls attention to this variety, including the use of computers to assist traditional observation, to estimate solutions to intractable sets of mathematical equations, and as simulating devices (see Humphreys 2004, 49-51 and chapter 4). So perhaps it also would be appropriate to say that the computer is an instrument for these sorts of purposes as well.

But why stop there? Consider the use of computers as simulating devices, i.e. for modeling the evolution of real or imagined systems. When used in this way, computers are claimed by philosophers and scientists alike to serve various epistemic and cognitive purposes. If so, then perhaps we can characterize the digital computer as an especially flexible instrument, useful for a broad range of purposes in the course of scientific research-a sort of Swiss Army knife of scientific instruments, with computation at its core. In this case, there may be quite a few answers to the question, what is the digital computer an instrument for? In what follows, I survey several common answers, limiting my analysis to computers in a simulating mode. I aim to illustrate the breadth of uncontroversial answers that have already been given and to call attention to a more contentious one that encourages reflection on the limits of what computer simulation models (and digital computers) can do. ${ }^{2}$

Exploring models. Sometimes computers are used in a simulating mode as a means of exploring the characteristics of models. For instance, we might propose to model the evolution of a real-world system with a set of differential equations that we cannot solve analytically for initial and boundary conditions of interest, leaving it unclear whether their solutions fit with the observed behavior of the system in expected or hoped-for ways. A computer might be used to repeatedly estimate solutions using numerical methods, producing a simulation whose results can be displayed on the computer's screen. What we see happening in the simulation may allow us to answer questions of interest about the modeling equations that we originally specified, e.g. whether their solutions do have expected or desired characteristics. Care must be taken when making such inferences from computer simulation results to modeling equations, however, since solutions estimated numerically using a finite-precision computer will differ a least a little bit from the corresponding exact solutions (see also Parker 2009). ${ }^{3}$

2 For each of the answers presented below, much more could be said; my emphasis is influenced by my past experience and is not intended to imply that work not discussed is unimportant.

3 These complications are avoided when the original model specifies equations that are 
Developing explanations. Computers are also used in a simulating mode for the purpose of developing explanations of observed phenomena. What we see happening in a simulation-available for visual inspection on a computer screen-may suggest a possible explanation of a phenomenon, or may help us to fill in gaps in an explanation that we have already started to sketch. In many cases, when computer simulation models are employed with such explanatory goals in mind, simulation results in effect serve as surrogate observational data, standing in for observational data that we have not been able to collect. For instance, it is difficult to observe in fine detail the evolution of conditions inside severe thunderstorms, but we can examine the results of high-resolution simulations to see what they suggest about that evolution; in practice, such simulations have played an important role in developing explanations of features of storm behavior (see Houze 1994, 294-95; Wilhelmson et al. 1990; Winsberg 1999). ${ }^{4}$

Forecasting. In addition, computer simulation models are used to forecast the behavior of real-world systems. Sometimes simulation results are interpreted directly as forecasts, while in other cases results go through some further processing. Perhaps the most familiar example of this use of computer simulation models is in forecasting the weather. A computer simulation model is initialized with an estimate of the state of the atmosphere at some recent time-typically that estimate is reached in a complicated way using both observational data and previous forecasts; the evolution of atmospheric conditions over a time period of interest is then simulated by running the model, producing results that subsequently undergo further adjustment in light of errors in previous simulations, before being offered as forecasts of the weather.

Designing and interpreting experiments. In high-energy physics, computer simulation models play an important role as instruments for experimental design and data interpretation. They are used to simulate what will happen when subatomic particles are made to collide at very high speeds in a detector; the collisions and the trajectories of their products, as well as the behavior of the detector, are simulated to reveal what data we can expect to collect in an experiment if our models of subatomic structure are correct. Decisions about which data channels to record in the experiment, and with what frequency, are made in light of such simulation results. Moreover, the raw data that are then collected in the experiment

discrete in time and can be solved exactly, as in the case of some agent-based models of social systems that specify rules for updating agent characteristics at each time step in light of the characteristics of nearby agents.

${ }^{4}$ Likewise, Bechtel and Abrahamsen (in press) provide an excellent discussion of how computational modeling can facilitate the development of mechanistic explanations of biological and cognitive phenomena. 
are interpreted using the simulated data as reference. As one physicist put it, "The simulation package is an intrinsic part of any high-energy experiment in as much as the detectors themselves; it is a mandatory component of any experiment from the design stage to the final result" (Perret-Gallix 2002, 488).

Measuring. Some philosophers suggest that computer simulation models can be instruments for measuring, too. Weaker and stronger versions of this view can be articulated. A weaker version says that some computer simulation models are able to serve the same function as measuring devices, i.e. they can reliably deliver information about real-world target systems that we might otherwise seek using traditional measuring devices. A stronger and more controversial version says that some computer simulation models are measuring instruments-their results constitute measurements of the properties of real-world target systems.

The latter view was defended recently by Margaret Morrison (2009), who sees important similarities between some computer simulation results and traditional measurements: both are model-dependent in various ways, and both involve causal contact with the real-world systems about which information is sought. ${ }^{5}$ The causal contact may be less straightforward in the case of computer simulation-grounded in the way simulated objects or processes stand in for particular real-world counterparts-but the differences do not prevent the classification of some simulation results as measurements. Morrison specifically has in mind the results of certain kinds of molecular dynamics simulations that use particle models, where "the particles in the simulation model can be directly identified with physical objects (via the theoretical model of the system)" (Morrison 2009, 45); the same type of theoretical model would be relied upon in carrying out more traditional measurements, e.g. in constructing the measuring apparatus and/or interpreting its readings.

There are various reasons why we might resist the view that computer simulation results can be measurements of the properties of real-world target systems. For instance, we might doubt that computer simulation studies (including the sort that Morrison discusses) really do involve the right sort of causal contact with the target system: if the measurement of a property had by a system at time $t$ must involve causal contact with the system at or after $t$, then it seems that computer simulation studies

${ }^{5}$ Something like the stronger view also seems to be advocated by Stephen Norton and Fred Suppe (2001), who argue that simulation models "provide an alternative technology for probing real-world phenomena" (99) and thus can be "another source of empirical data" (87). 
generally do not qualify. ${ }^{6,7}$ But I will not attempt to resolve the matter here. What is clear is that Morrison's discussion prompts us not only to consider the limits of what computer simulation models (and digital computers) can be instruments for but also to think more deeply about what is essential to genuine measurement, if anything. Doing so may lead us to a better understanding of both computer simulation and measurement.

To sum up, then, it seems we can give an impressive array of answers to the question, what is the digital computer an instrument for? When used in a simulating mode, the computer can be (at least) an instrument for exploring models, for developing explanations, for forecasting, for designing and interpreting experiments, and-some would argue-for measuring target system properties.

\author{
WENDY S. PARKER \\ Department of Philosophy \\ Ohio University \\ parkerw@ohio.edu
}

\title{
REFERENCES
}

Bechtel, William, and Adele Abrahamsen. (in press). Dynamic mechanistic explanation: Computational modeling of circadian rhythms as an exemplar for cognitive science. Studies in History and Philosophy of Science Part A. Houze, Robert. 1994. Cloud Dynamics. San Diego: Academic Press.

Humphreys, Paul. 2004. Extending Ourselves: Computational Science, Empiricism, and Scientific Method. New York: Oxford University Press.

Morrison, Margaret. 2009. Models, measurement and computer simulation: The changing face of experimentation. Philosophical Studies 143(1): 33-57.

6 The most straightforward way in which simulation studies might involve causal contact with the target system is via initial conditions, when these are obtained by traditional measurement. But since most computer simulations are run forward in time, this causal contact typically fails to meet the requirement specified above. It is unclear whether the sort of causal contact that Morrison has in mind meets the requirement.

7 It is interesting to note that there are studies involving computer simulation that do seem to meet the requirement. Atmospheric data assimilation, for instance, estimates the state of Earth's atmosphere at time $t$ using both simulation results for $t$ and observational data collected in a window of time extending on either side of $t$ (see e.g. Talagrand 1997). And intuitively the results of these studies are more plausibly classified as measurements. But typical computer simulation studies do not involve combining simulation results with observational data in the way that data assimilation does. 
Norton, Stephen, and Fred Suppe. 2001. Why atmospheric modeling is good science. In Changing the Atmosphere: Expert Knowledge and Environmental Governance, eds. Clark Miller and Paul N. Edwards, 67-105. Cambridge, MA: MIT Press.

Parker, Wendy S. 2009. Does matter really matter? Computer simulations, experiments and materiality. Synthese 169: 483-96.

Perrit-Gallix, Denis. 2002. Simulation and event generation in high-energy physics. Computer Physics Communications 147: 488-93.

Talagrand, Olivier. 1997. Assimilation of observations: An introduction. Journal of the Meteorological Society of Japan 75: 191-205.

Wilhelmson, Robert B., Brian F. Jewett, Crystal Shaw, Louis J. Wicker, Matthew Arrott, Mark Bajuk, Colleen Bushell, Jeffrey Thingvold, and Jeffery Yost. 1990. A study of the evolution of a numerically modeled severe storm. International Journal of Supercomputing Applications 4: 20-36.

Winsberg, Eric. 1999. Simulation and the philosophy of science: Computationally intensive studies of complex physical systems. PhD Dissertation, Indiana University. 